\title{
SHORT-TERM VARIATION OF THE EARTH'S ROTATION AND THE SOLAR ACTIVITY
}

D. DJUROVIC $(*)$ and P. PAQUET $(+)$

$\left.{ }^{*}\right)$ University of Belgrade, Inst. of Astronomy, Belgrade.

$(+)$ Royal Observatory of Belgium, Brussels.

\begin{abstract}
In 1980, Feissel et al. identified a quasi-cyclic variation of 55 days in the irregularities of the Earth Rotation (ER) later detected in the Atmospheric Angular Momentum (AAM) (Langley et al., 1981). The purpose of this work is to analyse whether the causes of this cycle could lie in the physical processes of the Sun. The Wolf Numbers (WN) are used as parameters of the solar activity. Their spectral analysis over the period 1967-1985 shows such a component at 51 days. Analysis of three other periods, among which is the MERIT campaign, confirms it as well as during low or increasing solar activity periods.
\end{abstract}

\section{INTRODUCTION}

Munk and McDonald (1960) discuss the problem of the solar wind and geomagnetic field interactions and their contribution to the variations of $\omega$, the angular velocity of the Earth. They concluded that these interactions may contribute to non-secular variations of $\omega$. On the other hand the investigations of Lambeck et al $(1973,1974,1976,1981)$, Okazaki $(1977$, 1979), Hide et al (1980) conclude that the atmospheric zonal circulation represents the dominant cause of the short-periodic and irregular variations of $\omega$; also Jacchia (1967) established the relationship between the solar radiation and the geomagnetic field and the air density variations. From their results Djurovic $(1981,1983)$ assumes that there exist common cyclic variations of solar activity, zonal component of the AAM, geomagnetic field and ER velocity. From this last paper it could be supposed that solar activity contributes to the variations of $\omega$, with the atmosphere as interface.

Besides the above mentioned papers, there are authors considering that solar activity does not contribute to the ER perturbations; see for example Schatzman (1966), Lambeck (1980).

Since the mechanism able to transmit the solar radiation energy to the ER is not well known, the problem of the relationships between solar activity and ER, strictly speaking, is still open today. However, it seems that the observational results are sufficient to found a hypothesis that such relationships exist. In favour of this are the results presented in this paper. This analysis is addressed to the short period, more precisely the variations around 55 days identified by Feissel et al (1980) as a quasi-cyclic variation of $\omega$, and later identified in the AAM zonal component (Langley et al., 1981). The idea that the origin of this cycle could lie in the physical processes of the Sun is re-inforced by an analysis presented in 
Pap (1985), in which the 55 day line of the spectra of projected areas of all observed spots appears also very clearly and is even more emphasized than the line corresponding to the Sun's rotation. The results given in this paper are based on the raw values of UT1-TAI series published in the BIH Annual Reports and on the series of daily Wolf Numbers (WN) as published by the Sunspot Index Data Center (SIDC).

\section{FOR 1967-1985, SHORT-TERM COMPONENTS IN THE WOLF NUMBERS AND UT1-TAI}

Over the period 1967-1985, as a first test, a spectral analysis using the Minimum Cross Entropy method has been applied on the residuals deduced by eliminating from the original WN data a smoothed function defined by Vondrak's method (1969) using a smoothing factor $\epsilon=10^{-9}$.

The resulting spectra is presented in Figure 1. A 51 day variation appears very clearly and the relative amplitude of this particular peak is $32 \%$ of the main peak observed at 27 days.

By comparison, the spectral analysis of the UT1-TAI data, covering the same period and from which the components greater than 6 months are removed, provides the spectrum also given in Figure 1. The 54 day peak is small with respect to the other and is part of a broadband domain, extended till 90 days, with a larger peak around 86 days.
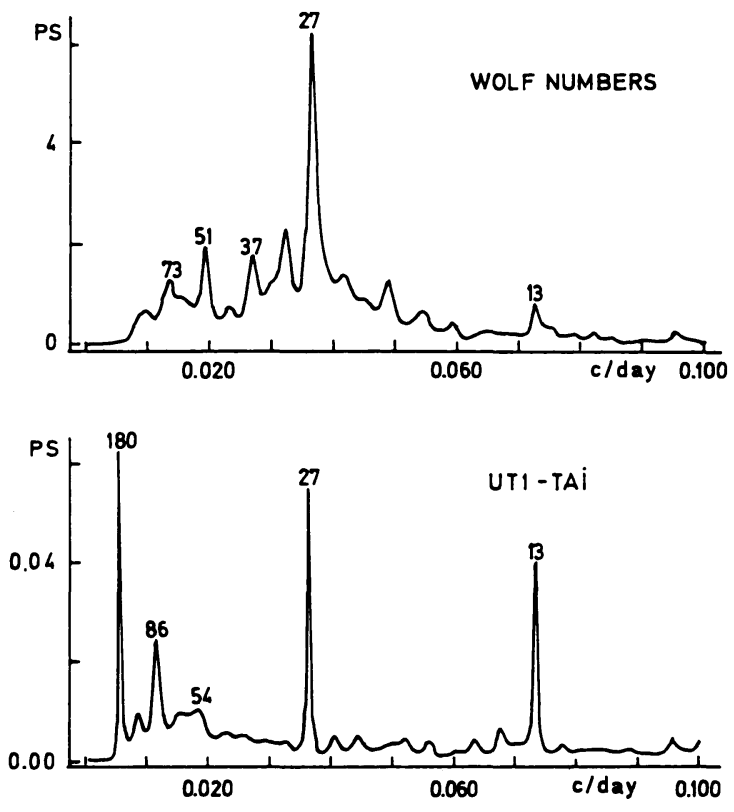

Figure 1.

Power spectrum of residuals of the Wolf Numbers and UT1TAI as as observed during the period 1967-1985 and filtered by the Vondrak's method $(\epsilon=$ $\left.10^{-7}\right)$ for UT1-TAI; $\left(\epsilon=10^{-8}\right)$ for the WN. 


\section{SHORT-TERM COMPONENT AT DIFFERENT PHASES OF SOLAR ACTIVITY}

The MERIT campaign having been a period during which the quality of the obervations were significantly increased, we started our analysis to check the presence of the 55 day component in the UT1-TAI series deduced by IPMS, from classical astronomical data only, and by NGS, from the processing of the VLBI data.

Aiming to isolate the short-term component, the two series were filtered by the Vondrak's method, the choice of the smoothing factor $\epsilon$ being fixed on the basis of the paper of Feissel et al (1984) and selected to eliminate, from the ER irregularities, the long-term components including the semi-annual one. With $\epsilon=10^{-6}$, the smoothed function contains the terms with periods over 100 days and a small part (15\%) of the 50 day variation. In consequence, the residuals contain several tidal terms and the major part (85\%) of the 50 day variation; the two sets of residuals are plotted in Figure 2 ( $a$ and $b$ ). On the same Figure 2, the continuous curves are smooth with $\epsilon=10^{-4}$, which contain part of the tidal terms $M_{m}$ and $M_{f}$ superposed on the 50 day variation. By a visual inspection of these smoothed curves it appears that 8 main cycles subdivide the whole period of 400 days. Spectral analysis of the residuals, derived from both VLBI and IPMS data clearly confirms the presence of the 55 day component, identified at 57 days during the MERIT campaign (Fig. 3). By reference to the main peak the relative amplitudes of the two others are $56 \%$ and $13 \%$.

The daily WN corresponding to the MERIT campaign were analysed, according to the same procedure applied to UT1-TAI data. From the original data the components greater than a year were removed by the Vondrak method $\left(\epsilon=10^{-9}\right)$ and the residuals contain the contribution of all components shorter than a year. The residuals, presented also in Figure 2(c), show a 55 day component as reflected by the continuous curve $\left(\epsilon=10^{-5}\right)$. It is confirmed by the spectral analysis (Figure 3 ) and with respect to the main peak observed at 29 days, the relative amplitude of the 55 day component is $66 \%$. The variation around 55 day is thus clearly identified in this particular record of the WN. However, as the MERIT campaign has been conducted close to the end of a solar activity cycle, two other periods were selected to confirm this possible relation of a 55 day component observed in both the WN and UT1-TAI series.

The two selected periods cover a weak solar activity period, from 22 Jan 1976 to 25 Sept 1977 and an increasing solar activity period, from $20 \mathrm{Dec} 1977$ to $15 \mathrm{Sept} 1980$. The WN and UT1-TAI data were filtered using the same smoothing degree as above. The associated results, given in Figure 4 as spectral density diagrams, confirm the presence of a variation in the solar activity close to 55 days.

\section{CORRELATION BETWEEN UT1-TAI AND WOLF NUMBERS VARIATIONS}

The maximum value of the coefficient of correlation between the smoothed curves associated with the IPMS and WN residuals is obtained with a phase shift of about 20 days; it reaches a value of 0.36 while the $t$-Student variable is equal to 3.47 ; it corresponds to a level of confidence of $99 \%$. 

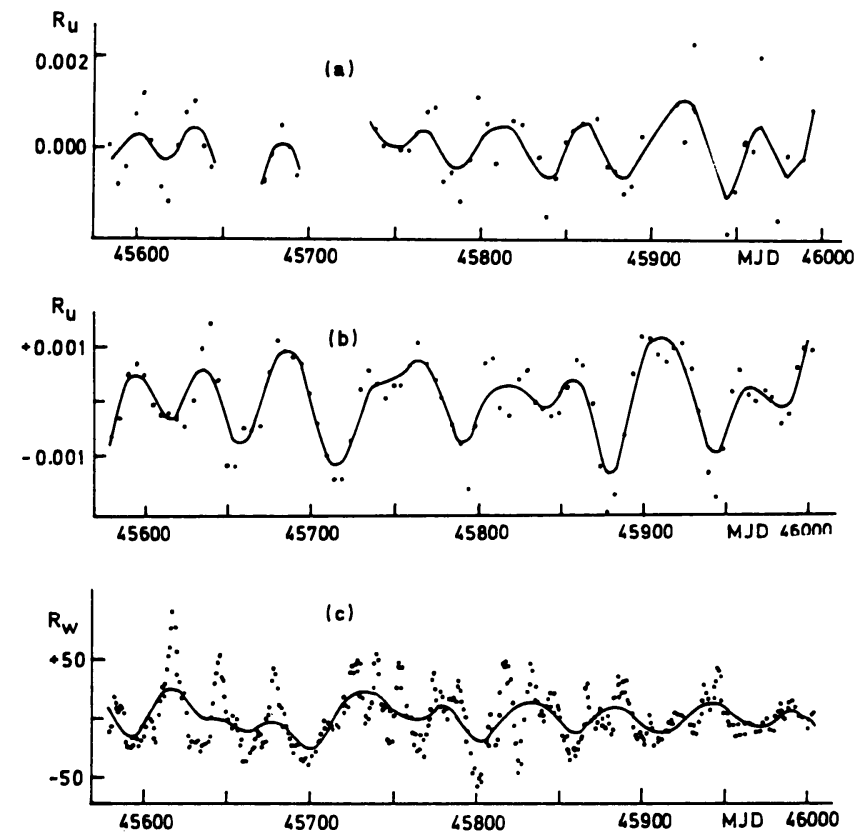

Figure 2. For the MERIT campaign, residuals of original data filtered to remove the long term components: (a) Related to UT1-TAI as observed by VLBI. (b) Related to UT1-TAI as deduced by IPMS (Astronomical data only). (c) Related to the Wolf Numbers.
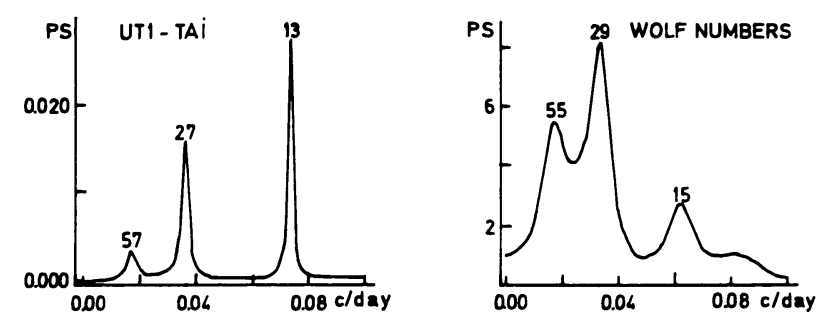

Figure 3. Spectral analysis of UT1-TAI and WN as observed during the MERIT campaign and filtered to remove long term components. 

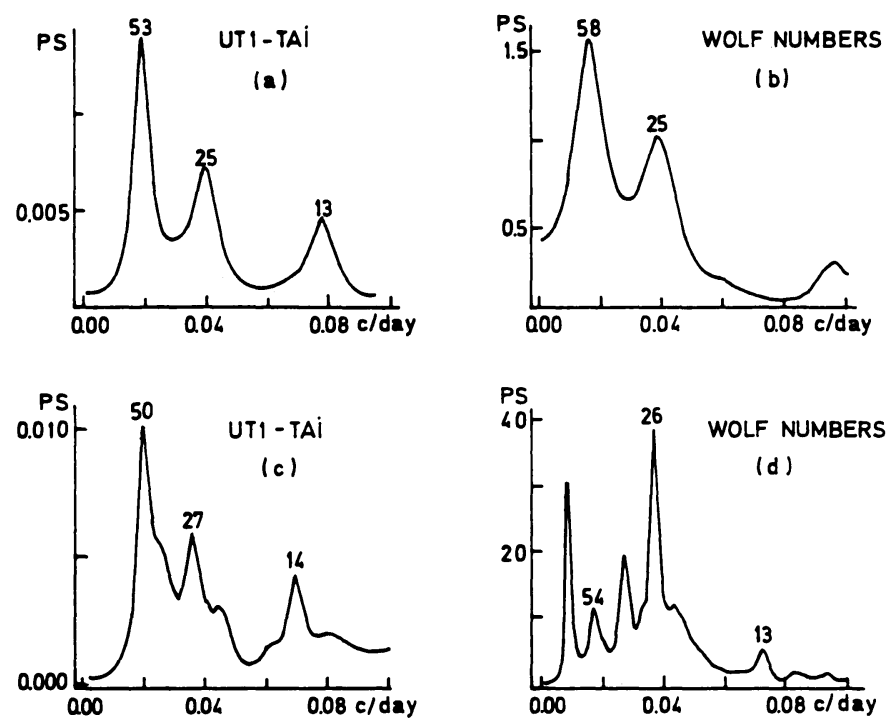

Figure 4. Spectral analysis of UT1-TAI and WN as observed during low and increasing solar activity periods. The original data were filtered to remove the long term components.

The same computation between the smoothed curves associated with the VLBI residuals (Fig. 2a) leads to the values of $0.40,3.09,99 \%$ respectively.

\section{CONCLUSION}

The existence of a 55 day variation in the solar activity is clearly established through the analysis of the daily Wolf numbers observed as well during low or increasing solar activity. For the same periods, UT1-TAI series confirm of course the accepted 55 day component in the ER variations.

The same numerical methods having been used to analyse all the series and this particular component of 55 day in the ER variation being known, lead us to believe that the corresponding component identified in the solar activity is real. The correlation between the two series is of the order of $99 \%$. 


\section{BIBLIOGRAPHY}

Djurovic D., 1981 Astron. Astrophys. 100, 156.

Djurovic D., 1983. Astron. Astrophys. 118, 26.

Feissel M., Gambis D., 1980. C. R. Acad. Sci. Paris, B271.

Feissel M., Lewandowski W., 1984. Bull. Geod., 58, 4.

Hide R., Birch N.T., Morrison L.V., Schea D.J., White A.A., 1980, Nature, 286.

Jacchia L.G., 1967. Solar Terrestrial Phys., 5.

Lambeck K., Cazenave A., 1973. Geophys. J. Roy. Astr. Soc. 32, 79

Lambeck K., Cazenave A., 1974. Geophys. J. Roy. Astr. Soc. 38, 49

Lambeck, K., 1980. The Earth's variable rotation. Cambridge Univ. Press.

Lambeck K., Hopgood P., 1981. Geophys. J. Roy. Astr. Soc. 64, 67.

Langley R.B., King R.W., Shapiro I.I., Rosen R.D., Salstein D.A., 1981. Nature, 294, 730.

Munk W.H., Mc Donald G.J.F., 1960. The rotation of the Earth. Cambridge Univ. Press.

Okazaki S., 1977. Publ. Astron. Soc. Japan 29, 619.

Okazaki S., 1979. Publ. Astron. Soc. Japan 31, 613.

Pap J., 1985. Solar Physics, 97, 21.

Schatzman E., 1966. The Earth-Moon System. ed. Marsden B. and Cameron A., Plenum Press, NY.

Vondrak J., 1969. Bull. Astron. Inst. Czechoslovakia, 20, 6.

\section{DISCUSSION}

Lambeck: Casenave and I looked at the $\simeq 50$ day oscillation in 1974 and I recall that it could be explained by a near-equatorial oscillation in the Pacific atmosphere and that this oscillation was not clearly seen elsewhere (from Julian and co-author). Is this regional oscillation consistent with it being externally driven as you suggest?

Reply by Rosen: A number of mechanisms have been proposed by meteorologists to explain this fluctuation. None of these seem to require changes in the solar constant on this time scale, but instead rely on internal instabilities in the earth-atmosphere-ocean system. 